University of Windsor

Scholarship at UWindsor

$7-1-2020$

\title{
The structures of polyunsaturated lipid bilayers by joint refinement of neutron and X-ray scattering data
}

\author{
Drew Marquardt \\ University of Windsor \\ Frederick A. Heberle \\ The University of Tennessee, Knoxville \\ Jianjun Pan \\ University of South Florida, Tampa \\ Xiaolin Cheng \\ The Ohio State University \\ Georg Pabst \\ Karl-Franzens-Universitat Graz
}

See next page for additional authors

Follow this and additional works at: https://scholar.uwindsor.ca/chemistrybiochemistrypub

Part of the Biochemistry, Biophysics, and Structural Biology Commons, and the Chemistry Commons

\section{Recommended Citation}

Marquardt, Drew; Heberle, Frederick A.; Pan, Jianjun; Cheng, Xiaolin; Pabst, Georg; Harroun, Thad A.; Kučerka, Norbert; and Katsaras, John. (2020). The structures of polyunsaturated lipid bilayers by joint refinement of neutron and X-ray scattering data. Chemistry and Physics of Lipids, 229.

https://scholar.uwindsor.ca/chemistrybiochemistrypub/149

This Article is brought to you for free and open access by the Department of Chemistry and Biochemistry at Scholarship at UWindsor. It has been accepted for inclusion in Chemistry and Biochemistry Publications by an authorized administrator of Scholarship at UWindsor. For more information, please contact scholarship@uwindsor.ca. 


\section{Authors}

Drew Marquardt, Frederick A. Heberle, Jianjun Pan, Xiaolin Cheng, Georg Pabst, Thad A. Harroun, Norbert Kučerka, and John Katsaras 


\title{
The structures of polyunsaturated lipid bilayers by joint refinement of neutron and X-ray scattering data
}

Drew Marquardt ${ }^{a, b *}$, Frederick A. Heberle ${ }^{c}$, Jianjun $\operatorname{Pan}^{d}$, Xiaolin Cheng ${ }^{e}$, Georg Pabst ${ }^{f}$, Thad A. Harroun ${ }^{g}$, Norbert Kučerka ${ }^{h}$, John Katsaras ${ }^{g i j *}$

${ }^{a}$ Department of Chemistry and Biochemistry, University of Windsor, Windsor, ON, Canada

${ }^{b}$ Department of Physics, University of Windsor, Windsor, ON, Canada

${ }^{c}$ Department of Chemistry, University of Tennessee, Knoxville, TN, 37996, USA

${ }^{d}$ Department of Physics, University of South Florida, Tampa, FL, USA

${ }^{e}$ Division of Medicinal Chemistry and Pharmacognosy, The Ohio State University, Columbus, OH, USA

${ }^{f}$ University of Graz, Institute of Molecular Biosciences, Biophysics Division, 8010 Graz, Austria

${ }^{g}$ Department of Physics, Brock University, St. Catharines, ON, Canada

${ }^{h}$ Joint Institute for Nuclear Research, Frank Laboratory of Neutron Physics, Dubna, Russia and Department of Physical Chemistry of Drugs, Faculty of Pharmacy, Comenius University in Bratislava, Slovakia

${ }^{i}$ The Bredesen Center, University of Tennessee, Knoxville, TN, USA

${ }^{j}$ Shull Wollan Center, Oak Ridge National Laboratory, Oak Ridge, TN, USA

${ }^{j}$ Large Scale Structures Group, Neutron Sciences Directorate, Oak Ridge National Laboratory, Oak Ridge, TN, USA

${ }^{k}$ Department of Physics and Astronomy, University of Tennessee, Knoxville, TN, USA

\begin{abstract}
We present the detailed structural analysis of polyunsaturated fatty acid-containing phospholipids namely, 1-palmitoyl-2-docosahexaenoyl-sn-glycero-3-phosphocholine (PDPC) and 1stearoyl-2-docosahexaenoyl-sn-glycero-3-phosphocholine (SDPC). A newly developed molecular dynamics (MD) simulation parsing scheme for lipids containing fatty acids with multiple double bonds was implemented into the scattering density profile (SDP) model to simultaneously refine differently contrasted neutron and X-ray scattering data. SDP analyses of scattering data at $30{ }^{\circ} \mathrm{C}$ yielded lipid areas of $71.1 \AA^{2}$ and $70.4 \AA^{2}$ for PDPC and SDPC bilayers, respectively, and a model free analysis of PDPC at $30{ }^{\circ} \mathrm{C}$ resulted in a lipid area of $72 \AA^{2}$. In addition to bilayer structural parameters, using area-constrained MD simulations we determined the area compressibility modulus, $K_{A}$, to be $246.4 \mathrm{mN} / \mathrm{m}$, a value similar to other neutral phospholipids.
\end{abstract}


Keywords: polyunsaturated fatty acids, neutron scattering, X-ray scattering, MD simulations

\section{Introduction}

Phospholipids that contain polyunsaturated fatty acids (PUFAs) are a special class of lipids that constitute a biologically influential group of biomolecules essential for normal growth and development. [1] Recently, PUFA-containing phospholipids have attracted increased attention because they have been shown to be essential for a range of cellular functions [2] and for their structural and functional roles in membranes. [3] PUFAs are generally classified as omega-3 or omega-6 fatty acids, depending on the location of the double bond in relation to the acyl chain's terminal methyl group (i.e., omega-3 has its last double bond located three atoms from the terminal methyl group). The dietary consumption of omega-3 fatty acids is known to alleviate chronic health conditions including hypertension, diabetes, and arthritis $[4,5]$, while a diet rich in omega- 6 fatty acids is linked to increased blood viscosity, vasospasm, and vasoconstriction. [6] However, there is increasing evidence that both PUFAs are needed for maintaining good health [7] and an imbalance results in diseases such as diabetes. [5]

PUFAs associated with phospholipids exhibit increased dynamics compared to their saturated analogues. For example, the low energy barrier for rotation about C-C single bonds between olefanic and aliphatic carbons in PUFAs allows for sub-nanosecond conformational transitions, resulting in a much higher degree of chain disorder, which increases the interaction probability between PUFAs and the lipid headgroup. [8] In the case of docosahexaenoic acid (DHA; 22:6) containing lipids, it has been shown that DHA explores its entire conformational space in $\sim 50$ ns. [9] The high degree of PUFA chain disorder also results in thinner bilayers. An example is diarachidonoyl PC (DAPC; di20:4PC), which has an acyl chain thickness comparable to 1,2-dilauroyl-sn-glycero-3-phosphocholine (DLPC; di12:0PC), even though DAPC has eight more methines per hydrocarbon chain. [10,11] However, a more telling example of PUFA chain disorder is given by their segmental order parameters $\left(S_{C D}\right)$. Both simulation [12] and experimental results $[13,14]$ show reduced $S_{C D}$ values $\left(S_{C D}<0.05\right)$ along the length of DHA chains in SDPC bilayers as a result of increased 
mobility associated with their double bonds. Moreover, mixed chain PUFA PCs, such as 1palmitoyl-2-arachidonoyl-sn-glycero-3-phosphocholine (PAPC; 16:0-20:4PC) and 1-stearoyl2-docosahexaenoyl-sn-glycero-3-phosphocholine (SDPC; 18:0-22:6PC), have been reported to form thinner bilayers with larger areas per lipid, compared to bilayers whose lipids contain either a saturated or mono-unsaturated fatty acid chain in their sn-2 position. In addition to their high mobility, PUFAs are susceptible to reactive oxygen species. Their vulnerability to oxidation creates unique biological problems, both structurally and in terms of biological availability. Structurally, PUFA oxidation products can alter the physical properties of a bilayer, which can ultimately lead to the malfunction of membrane associated proteins.

Although not common, PUFA-containing phospholipid simulations do exist. However, it appears that in some cases the existing force fields result in physically unrealistic values. For example, Klauda et al. reported a headgroup-headgroup spacing $\left(D_{H H}\right)$ for DAPC [15] that is similar to the $D_{H H}$ of POPC $(36 \AA)$, [16] but much larger than the experimentally determined value. $[10,17]$ Inconsistencies between experiment and simulations thus pose significant problems when trying to accurately explain the physical behavior of PUFA containing phospholipids.

Here, we present a scattering density profile (SDP) model for lipids with PUFAs, namely the mixed chain lipids 1-palmitoyl-2-docosahexaenoyl-sn-glycero-3-phosphocholine (PDPC; 16:0-22:6PC) and 1-stearoyl-2-docosahexaenoyl-sn-glycero-3-phosphocholine (SDPC; 18:022:6PC). Combining small angle X-ray scattering (SAXS), small angle neutron scattering (SANS), and molecular dynamics (MD) simulations we determined their bilayer structures with a high degree of accuracy and unprecedented spatial resolution.

\section{Materials and Methods}

\subsection{Materials}

Synthetic 1-palmitoyl-2-docosahexaenoyl-sn-glycero-3-phosphocholine (PDPC) and 1-stearoyl2-docosahexaenoyl-sn-glycero-3-phosphocholine (SDPC) were purchased from Avanti Polar (Alabaster, AL) lipids as chloroform solutions and used as received. Ultrapure $\mathrm{H}_{2} \mathrm{O}$ was obtained from a High-Q purification system (Wilmette, IL), and 99.9\% $\mathrm{D}_{2} \mathrm{O}$ was purchased from Cambridge Isotopes (Andover, MA). 


\subsection{Vesicle preparation}

Large unilamellar vesicles (LUVs) were prepared using previously established procedures. [16, 18] In short, PDPC films were prepared by transferring the desired volumes of stock lipid solutions to glass vials (Hamilton USA, Reno, NV) and then evaporating the organic solvent using a combination of Ar gas and gentle heating, followed by drying in vacuo for no less than 4 hours. Samples were then hydrated with $100 \% \mathrm{D}_{2} \mathrm{O}$ for SANS measurements, or ultrapure $\mathrm{H}_{2} \mathrm{O}$ for SAXS measurements, using a series of 7 freeze-thaw-vortex cycles. The lipid dispersions were extruded using an Avanti mini-extruder with a $50 \mathrm{~nm}$ diameter pore size polycarbonate filter. The SANS sample was then divided into three aliquots and diluted to the desired external contrast condition (100, 75 and $\left.50 \% \mathrm{D}_{2} \mathrm{O}\right)$ using an appropriate amount of $\mathrm{H}_{2} \mathrm{O}$. The lipid concentration used for SAXS and SANS measurements was $\sim 12 \mathrm{mg} / \mathrm{ml}$. To minimize lipid oxidation, all sample manipulations were performed in a Techni-Dome $360^{\circ}$ Glove Box Chamber under an Ar atmosphere Oxygen was measured with an Oxy-Sen oxygen monitor (Alpha Omega Instruments, Cumberland, R.I.) and maintained below $2 \%$ for all sample manipulations. Aliquots of the PUFA samples were taken before and after SANS measurements to evaluate oxidative damage by UV/Vis spectroscopy. UV/Vis experiments were conducted with an Ocean Optics USB4000 CCD array detector coupled to an RF deuterium source with a tungsten halogen bulb (Mississauga, Canada) using the procedure described by Marquardt et al. [10]

\subsection{Density measurements}

Lipid dispersions for volume measurements were prepared by hydrating $\sim 25 \mathrm{mg}$ of PDPC or SDPC with $\sim 1.5 \mathrm{~g}$ of degassed ultrapure $\mathrm{H}_{2} \mathrm{O}$, followed by gentle sonication at room temperature until the dispersion was uniformly "milky". Care was taken to avoid exposure of the sample to oxygen, thus sample manipulations were carried out in an Ar atmosphere $\left(\mathrm{O}_{2}<2 \%\right)$. The temperature dependent densities of water $\left(\rho_{w}\right)$ and of the lipid dispersions $\left(\rho_{s}\right)$ were determined by a temperature-controlled Anton-Paar DMA5000 (Graz, Austria) vibrating tube densitometer. The lipid volume $\left(V_{L}\right)$, at a given temperature, was calculated as previously described [19, 20, 21] 


$$
V_{L}=\frac{M W_{L}}{N_{A} \rho_{s}}\left[1+\frac{m_{w}}{m_{s}}\left(1-\frac{\rho_{s}}{\rho_{w}}\right)\right]
$$

where $M W_{L}$ is the molecular weight of the lipid, $N_{A}$ is Avogadro's number, and $m_{L}$ and $m_{W}$ are the masses of the lipid and water, respectively.

\subsection{Small-angle neutron and X-ray scattering}

X-ray data were taken at the Cornell High Energy Synchrotron Source (CHESS) G-1 station. $1.18 \AA$ wavelength X-ray photons were detected using a $1024 \times 1024$ pixel array FLICAM charge-coupled device (CCD) with $71 \mu \mathrm{m}$ linear dimension pixels. Samples were taken up in $1 \mathrm{~mm}$ quartz capillaries placed in a homemade temperature-controlled, multiple position sample holder. Neutron scattering experiments were performed at the High Flux Isotope Reactor (HFIR) CG-3 Bio-SANS instrument located at Oak Ridge National Laboratory (ORNL). ULV suspensions were loaded into $2 \mathrm{~mm}$ path-length quartz banjo cells (Hellma USA, Plainview, NY), sealed under an Ar atmosphere, and mounted in a temperature-controlled cell holder. Data were taken at a sample-to-detector distance (SDD) of 1.7 meters using $6 \AA$ wavelength neutrons $(\Delta \lambda / \lambda=0.15)$, resulting in a total scattering vector of $0.02<\mathrm{q}<0.3 \AA^{-1}$. Scattered neutrons were collected using a $192 \times 192$ pixel two-dimensional $(1 \mathrm{~m} \times 1 \mathrm{~m}){ }^{3} \mathrm{He}$ position-sensitive detector (ORDELA, Inc., Oak Ridge, TN). Two-dimensional data were reduced into a one-dimensional scattering intensity (I) vs the scattering vector (q) plot using ORNL's MANTID software. [22]

\subsection{Molecular dynamics simulations}

The CHARMM-GUI Membrane Builder [23] was used to generate coordinates for a PDPC bilayer containing a total of 200 lipids. Lipid hydrogen atoms were explicitly included (allatom model), including 6962 water molecules. MD simulations were performed using the NAMD 2.9 [24] and CHARMM 36 lipid force fields. [25] Periodic boundary conditions were applied and for each system, energy was minimized using the conjugated gradient algorithm for 5000 steps, followed by 2 ns of equilibration in a constant particle number, pressure, and temperature (NPT) ensemble. Equilibrium was determined by monitoring the system's area per lipid and its root-mean-square deviation (RMSD). In all simulations, the van der 
Waals (vdW) interactions were truncated via a potential-based switching function used by X-PLOR. Starting from a switching distance of $8 \AA$, the vdW force was brought smoothly to zero at the cut-off distance of $12 \AA$. Electrostatic interactions were treated using the particlemesh Ewald (PME) method with a $1.0 \AA$ grid spacing. [26] The r-RESPA multiple-time-step method [27] was employed with a 2 fs time step for bonded, and 2 and 4 fs time steps for short-range nonbonded and long-range electrostatic interactions, respectively. Bonds between hydrogens and other atoms were constrained using the SHAKE algorithm. [28]

We first simulated the PDPC bilayer using the NPT ensemble for 80 ns. Langevin dynamics were used to maintain a constant temperature of $303 \mathrm{~K}$, while the Nose-Hoover Langevin-piston algorithm $[29,30]$ was used to maintain a constant pressure of 1 bar. The z-axis was allowed to expand and contract independently of the $\mathrm{x}-\mathrm{y}$ plane (semi-isotropic pressure coupling). The resulting equilibrated lipid area was $63.5 \AA^{2}$. This simulation was then used to guide the development of an SDP model for the joint analysis of the SAXS and SANS data that resulted in an area per lipid of $70.2 \AA^{2}$. An additional set of 7 constant particle number, area, normal pressure, and temperature (NAPnT) simulations was performed, where the average area per lipid was constrained to $64,66,68,70,72,74$, and $76 \AA^{2}$, while the z-axis was allowed to expand and contract in order to maintain a constant Pn. Starting configurations for these simulations were selected snapshots from the NPT trajectory with lipid areas set close to their target values. The production run length for each of these simulations was between 96 and 128 ns, of which only the final 50 ns of each trajectory were used for data analysis. For each of the area-constrained simulations the surface tension, $\gamma$, was calculated from the difference between the normal and lateral components of the pressure tensor. [31,32] The lateral area compressibility modulus, $K_{A}$, was given by $K_{A}=\partial \gamma / \partial(\ln A)$. [33] All simulations were conducted on the Hopper supercomputer located at the National Energy Research Scientific Computing Center (NERSC).

\section{Results and Discussion}

Sample Integrity PUFA's are extremely sensitive to oxidative damage. Thus, to determine if any oxidative damage occurred over the course of a given measurement, we performed UV/Vis measurements. The majority of naturally occurring carbon-based free radicals are 
formed from the alkyl and allylic carbons in the lipid acyl chains. Allylic carbon radicals quickly isomerize, conjugating the double bonds in PUFAs, and produces the electron- $\pi$ system that absorbs UV light. After SANS experimentation, small amounts of oxidized lipids were detected as a small increase in absorbance around $250 \mathrm{~nm}$ (Fig. 1, red curve), compared to a fresh lipid preparation (Fig. 1, blue curve). However, when comparing samples that underwent experimentation to those exposed intentionally to air (Fig. 1, yellow and red curves, respectively), the amount of oxidative damage incurred by the SANS samples was negligible. Moreover, successive frames of SAXS data did not show any signs of sample degradation.

Insight from Area Constrained MD Simulations Simulations were performed using lipid areas ranging from 64.0 to $76.0 \AA^{2}$, generating a series of neutron and X-ray form factors for each system. Bilayer form factors were generated by computing the number density distribution of each atom from the simulation trajectories. [34] Electron density and neutron scattering length density profiles were calculated by summing the product of the number density of each atom with its number of electrons or neutron scattering length, respectively. The corresponding simulated X-ray and neutron scattering form factors were calculated from the Fourier transform of the solvent-subtracted ED or NSLD profile, as was done previously. $[25,35,36,37]$ The quality of the model-free analysis compares the calculated form factors with those from experiment, where the agreement was quantified by a reduced $\chi^{2}$ defined as:

$$
\chi^{2}=\frac{1}{N_{q}-1} \sum_{i=1}^{N}\left(\frac{\left|F_{s}\left(q_{i}\right)\right|-k \times\left|F_{e}\left(q_{i}\right)\right|}{k \times \Delta F_{e}\left(q_{i}\right)}\right)^{2},
$$

where $N_{q}$ is the number of experimental q-values (data points), $F_{s}$ and $F_{e}$ are the simulated and experimental form factors, respectively, $\Delta F_{e}$ is the experimental uncertainty, and $k$ is a scaling factor used to minimize $\chi^{2}$.

Figure 2 displays the reduced neutron, X-ray, and overall $\chi^{2}$ as a function of simulated area per lipid for PDPC at $30^{\circ} \mathrm{C}$ measured at three different SANS contrasts (i.e., 100, 75 and $50 \% \mathrm{D}_{2} \mathrm{O}$ ) and one SAXS contrast. Both the neutron and the overall $\chi^{2}$ values are at a minimum for an area per lipid of $72.0 \AA^{2}$, whereas the reduced X-ray $\chi^{2}$ decreases monotonically as the area per lipid increases. Figure 3 shows the model-free comparison 
between experimental and simulated form factors for the PDPC bilayer with an area per lipid constrained to $72.0 \AA^{2}$. Although the neutron data are in good agreement, this is not the case for the X-ray form factors, especially at the minimum position near $0.3 \AA^{-1}$ that relates to the bilayer thickness. Since the minimum position of the simulated X-ray form factor occurs at a smaller scattering vector q than the experimental X-ray form factor, we can surmise that the simulated bilayer lipid area of $72 \AA^{2}$ is too small. This observation partially explains why the X-ray reduced $\chi^{2}$ becomes smaller when the simulation lipid area increases (and the bilayer thickness decreases). Collectively, Figs. 2 and 3 suggest that the simulated bilayer at $A=72 \AA^{2}$ can qualitatively reproduce experimental scattering data (especially neutron form factors), whereas further tuning of the force field is required to better match the X-ray data and the area per lipid predicted by our SDP model, something that has been noted previously and highlights unresolved issues with simulations [38]. It should be pointed out that the fit to the neutron data leads to $A$ directly (considering only the total molecular volume obtained densitometrically), whereas the X-ray data are sensitive to the values of $D_{H H}$ and $D_{H 1}$ from the simulation. The simulations presented here provide nevertheless an important basis for the SDP model described below.

Lateral bilayer area compressibility Similar to the work of Waheed and Edholm [39], we used a series of MD simulations to determine the area compressibility modulus from the surface tension at several different lipid areas. Figure 4 shows the calculated $\gamma$ of PDPC bilayers at different lipid areas. A linear fit to the data yields an area compressibility modulus of $246.4 \mathrm{mN} / \mathrm{m}$, a value consistent with $K_{A}$ values of other PC lipids [40,33], and in good agreement with previously determined $K_{A}$ values for PUFAs [40].

SDP Model for PDPC and SDPC Previous SDP models for phospholipid containing mono-unsaturated fatty chains divided the acyl chains into terminal methyl $\left(\mathrm{CH}_{3}\right)$, methine $(\mathrm{CH})$, and methylene $\left(\mathrm{CH}_{2}\right)$ groups. [41, 42, 16, 36] Here, we adopted a similar parsing scheme for PDPC, except for the methine groups. Due to the increased number of olefinic carbons in docosahexaenoyl chains, our modified parsing scheme groups three $\mathrm{CH}$ groups into one component, represented by a Gaussian function, as shown in Figure 4 (CHa and $\mathrm{CHb}$ for a total of two components). For the headgroup, we used the same parsing scheme as previous SDP PC models. [41, 42, 16], with one exception. Previous SDP analyses of PC 
lipids used a $V_{H L}$ of $331 \AA^{3}$, compared to the $320 \AA^{3}$ we used here and which we form the area-constrained PDPC bilayer simulations ( $A=72.0 \AA^{2}$, please see above). This value for $V_{H L}$ is also consistent with previous experimental headgroup volume measurements [43] and using $V_{H L}$ values of $331 \AA^{3}$ and $320 \AA^{3}$ results in PUFA areas per lipid that differ by $\sim 0.5$ $\AA^{2}$, a difference that is considerably smaller than the experimental uncertainty $\left(\sim 1.5 \AA^{2}\right)$ that we determined for the final PDPC area. Similar to our previous SDP model-based analyses $[41,37,16,35,36]$, certain parameters were constrained to enhance the robustness of the fits to the data. Specifically, in addition to $V_{H L}$, the experimentally determined $V_{L}$ was fixed and soft constraints were applied to a group of parameters, whereby any deviation from the target values determined from MD simulations resulted in a quadratic penalty to the overall $\chi^{2}$ - soft-constrained parameters are denotated by an asterisk $(*)$. Finally, the distance between two $\mathrm{CH}$ components (i.e., $z_{C H b}-z_{C H a}$ ) was constrained. In the absence of such constraint the $\mathrm{CHs}$ tended to move in opposite directions, resulting in unphysical distances between the two moieties.

Joint SANS/SAXS analysis An SDP example used to jointly refine PDPC bilayer SANS and SAXS data at $30^{\circ} \mathrm{C}$ is shown in Figure 5. The volume probability of each component (Fig. 5E) is scaled by the component electron number and neutron scattering length, respectively, to generate the ED (Fig. 5C) and NSLD (Fig. 5D) profiles. The X-ray (Fig. 5B) and neutron (Fig. 5A) form factors are the Fourier transform of the total ED and NSLD profiles (after subtracting for bulk water), respectively. Results of these fits are presented in Table 1 for PDPC at $20^{\circ} \mathrm{C}, 30^{\circ} \mathrm{C}$, and $40^{\circ} \mathrm{C}$, as well as for SDPC at $30^{\circ} \mathrm{C}$. Table 1 also compares structural parameters obtained from the area-constrained MD simulations.

An important parameter commonly used to describe lipid bilayer structures is $A$, which is determined through a combination of lipid volumetric measurements and the bilayer thickness. Specifically, $A$ is related to the overall bilayer thickness, $D_{B}$, through the relationship $A=2 V_{L} / D_{B}$, where $V_{L}$ is the total lipid volume and $D_{B}$ is the Luzzati bilayer thickness, i.e., the Gibbs dividing surface found between the bilayer and water [44]. Alternatively, $A$ can be calculated based on the hydrocarbon chain thickness $D_{C}$, which corresponds to the interface between polar/nonpolar parts, i.e., $A=V_{H C} / D_{C}$. Other structural parameters listed in Table 1 are the headgroup-to-headgroup distance, $D_{H H}$, and the distance between 
the glycerol and phosphate groups, $D_{H 1}$. Finally, $\sigma_{H C}$ is the width of the error function describing the total hydrocarbon chain region, $z_{\mathrm{CH} i}$ and $\sigma_{\mathrm{CH} i}$ are the Gaussian center and width of the components (CHa, CHb, CH3, G1, G2 and G3). $r_{C H}$ and $r_{C H 3}$ are the volume ratios of $V_{C H} / V_{C H 2}$ and $V_{C H 3} / V_{C H 2}$, where $V_{C H}, V_{C H 2}$, and $V_{C H 3}$ refer to the average volumes of the $\mathrm{CH}, \mathrm{CH} 2$ and $\mathrm{CH} 3$ components, respectively, and $r_{G 1}$ and $r_{G 2}$ are the volume fractions, respectively, of the G1 and G2 components with respect to the total headgroup volume.

SDP analysis of PDPC bilayers at $30{ }^{\circ} \mathrm{C}$ resulted in an area per lipid of $71.1 \AA^{2}$, in good agreement with the $72.0 \AA^{2}$ determined by MD simulations (Table 1). This difference is mainly due to the following: (i) $D_{B}$, which in conjunction with $V_{L}$ determines the area per lipid, is more accurately obtained from SANS data; and (ii) the reduced SANS $\chi^{2}$ value is smallest at $72.0 \AA^{2}$ (i.e., the simulation of neutron form factors at $72.0 \AA^{2}$ agree best with the experimental data). The discrepancy between MD data and the X-ray form factor (Fig. 3) is reflected by the difference in $D_{H H}$ values between the simulated bilayer and the SDP model prediction. The larger $D_{H H}$ value from the simulated bilayer indicates that simulations with smaller $D_{H H}$ will agree better with the experimental X-ray form factor. (Note that $D_{H H}$ is defined by the maxima in the ED profile.)

Compared to previously reported SDP data for mixed chain lipids, the area per lipid value for PDPC at $30{ }^{\circ} \mathrm{C}$ is $\sim 7 \AA^{2}$ larger than that of POPC lipids (i.e., $64.3 \AA^{2}$ ). [16] Consistent with the lipid area result, the hydrocarbon chain thickness, $2 D_{C}$, of PDPC bilayers is $\sim 1 \AA$ smaller than that of POPC bilayers. [16] The values for $A$ and $2 D_{C}$ for PDPC are consistent with a disordered $s n-2$ PUFA chain. Not surprisingly, a monotonically increasing area per lipid with increasing temperature observed for PDPC is consistent with what has been reported previously. [16] Similarly, area per lipid of SDPC is $\sim 4.9 \AA^{2}$ larger than its mono-unsaturated analogue SOPC (i.e., $65.5 \AA^{2}$ ) [16], along with its associated invariance of $2 D_{C}$. Interestingly, $A$ for SDPC is identical to PDPC, within experimental error. That is, exchanging the palmitoyl fatty acid chain with stearoyl does not affect lipid packing, as this bilayer property is dominated by the presence of the disordered docosahexaenoyl acyl chain at the $s n-2$ position. Unfortunately, a direct comparison of PDPC and SDPC with their fully saturated analogues is not possible at these temperatures as di-palmitoyl PC and di-stearoyl 
PC only exist in the gel phase at the reported temperatures. The other thickness parameters associated with PDPC and SDPC are notably smaller than their mono-unsaturated analogues; i.e., $D_{B}$ and $D_{H H}$ are $\sim 2 \AA$ and $\sim 4 \AA$ smaller, respectively. This observation is consistent with the notion of the PUFA chain "snorkeling" up to the lipid headgroup region. The PUFA chains reside at the lipid-water interface an appreciable amount of time, thereby necessitating that the headgroups remain in its tilted orientation in order to shield the PUFA (umbrella model) [45] from the aqueous solvent, similar to what has previously been seen with POPC and SOPC bilayers. [46] It is worth noting that the dependency of area per lipid on acyl chain length is sensitive to the unsaturation and position of double bonds, mismatch in the unsaturation and length of the two chains, and likely to other structural characteristics of lipids. [47, 48] For example, the area decrease with increasing chain length was reported for di-saturated PCs, while the elongation of saturated chain in the case of saturated/mono-unsaturated lipids resulted in the area increase.[11]

Subtle but important differences in lipid bilayer structure induced by the presence of a PUFA chain, rather than a mono-unsaturated chain, can have profound biological implications. The transverse structure of a membrane has implications associated with integral protein stability, enzyme activation, and modulating charge-membrane interactions. [49] These results are yet another example of the importance of lipid diversity and how different lipid species affect the transmembrane structure.

\section{Summary}

We combined MD simulations with differently contrasted SANS and SAXS data to determine the structure of the PUFA containing phospholipids PDPC and SDPC with a high degree of structural detail. Simulations guided a model-based analysis of the experimental data that resulted in an area per lipid of $71.1 \AA^{2}$ for PDPC at $30{ }^{\circ} \mathrm{C}$. This result was supported by model free evaluation of PDPC bilayers, where simulations with different fixed areas were directly compared to experimental data and which yielded an area per PDPC of $72.0 \AA^{2}$. Future work will make use of the current SDP model and strategy to determine the bilayer structures for other commonly studied PUFA containing phospholipids. Importantly, the discrepancy between the different contrast scattering and simulations data, considering 
that the neutron and X-ray data sets were obtained using the same LUVs (prepared using the sample method), emphasizes the need for the further refinement of the MD simulation force fields.

\section{Acknowledgments}

SANS data were collected at the Bio-SANS instrument located at the High Flux Isotope Reactor, a DOE Office of Science User Facility operated by the Oak Ridge National Laboratory. The authors also wish to acknowledge personnel support from the Center for Structural Molecular Biology. J.K. is supported through the Scientific User Facilities Division of the Department of Energy (DOE) Office of Science, sponsored by the Basic Energy Science (BES) Program, DOE Office of Science, under Contract No. DEAC05-00OR22725. F.A.H acknowledges support from National Science Foundation grant No. MCB-1817929. D.M. acknowledges the support of the Natural Sciences and Engineering Research Council of Canada (NSERC), [funding reference number RGPIN-2018-04841].

\section{References}

[1] D. L. O’Connor, R. Hall, D. Adamkin, N. Auestad, M. Castillo, W. E. Connor, S. L. Connor, K. Fitzgerald, S. Groh-Wargo, E. E. Hartmann, J. Jacobs, J. Janowsky, A. Lucas, D. Margeson, P. Mena, M. Neuringer, M. Nesin, L. Singer, T. Stephenson, J. Szabo, V. Zemon, Growth and Development in Preterm Infants Fed Long-Chain Polyunsaturated Fatty Acids: A Prospective, Randomized Controlled Trial, PEDIATRICS 108 (2001) 359-371. doi:10.1542/peds.108.2.359.

[2] J. Hamilton, R. Greiner, N. Salem, H.-Y. Kim, n-3 Fatty acid deficiency decreases phosphatidylserine accumulation selectively in neuronal tissues, Lipids 35 (2000) 863869. doi:10.1007/S11745-000-0595-X.

[3] A. Catalá, Lipid peroxidation modifies the picture of membranes from the "Fluid Mosaic Model" to the "Lipid Whisker Model", Biochimie 94 (2012) 101-109. doi:10.1016/j.biochi.2011.09.025. 
[4] W. Stillwell, S. R. Wassall, Docosahexaenoic acid: membrane properties of a unique fatty acid, Chemistry and Physics of Lipids 126 (2003) 1-27. doi:10.1016/S00093084(03)00101-4.

[5] A. P. Simopoulos, Human Requirement for N-3 Polyunsaturated Fatty Acids, Poultry Science 79 (2000) 961-970. doi:10.1093/ps/79.7.961.

[6] A. P. Simopoulos, Essential fatty acids in health and chronic disease, The American Journal of Clinical Nutrition 70 (1999) 560s-569s. doi:10.1093/ajcn/70.3.560s.

[7] A. P. Simopoulos, Omega-6/Omega-3 Essential Fatty Acid Ratio and Chronic Diseases, Food Reviews International 20 (2004) 77-90. doi:10.1081/FRI-120028831.

[8] N. V. Eldho, S. E. Feller, S. Tristram-Nagle, I. V. Polozov, K. Gawrisch, Polyunsaturated Docosahexaenoic vs Docosapentaenoic AcidDifferences in Lipid Matrix Properties from the Loss of One Double Bond, Journal of the American Chemical Society 125 (2003) 6409-6421. doi:10.1021/ja029029o.

[9] O. Soubias, K. Gawrisch, Docosahexaenoyl Chains Isomerize on the Sub-Nanosecond Time Scale, Journal of the American Chemical Society 129 (2007) 6678-6679. doi:10.1021/ja068856c.

[10] D. Marquardt, J. A. Williams, N. Kučerka, J. Atkinson, S. R. Wassall, J. Katsaras, T. A. Harroun, Tocopherol Activity Correlates with Its Location in a Membrane: A New Perspective on the Antioxidant Vitamin E, Journal of the American Chemical Society 135 (2013) 7523-7533. doi:10.1021/ja312665r.

[11] T. A. Harroun, J. Katsaras, S. R. Wassall, Cholesterol Hydroxyl Group Is Found To Reside in the Center of a Polyunsaturated Lipid Membrane, Biochemistry 45 (2006) 1227-1233. doi:10.1021/bi0520840.

[12] X. Leng, J. J. Kinnun, D. Marquardt, M. Ghefli, N. Kučerka, J. Katsaras, J. Atkinson, T. A. Harroun, S. E. Feller, S. R. Wassall, $\alpha$-Tocopherol Is Well Designed to Protect Polyunsaturated Phospholipids: MD Simulations, Biophysical Journal 109 (2015) 16081618. doi:10.1016/j.bpj.2015.08.032. 
[13] S. E. Feller, K. Gawrisch, A. D. MacKerell, Polyunsaturated Fatty Acids in Lipid Bilayers: Intrinsic and Environmental Contributions to Their Unique Physical Properties, Journal of the American Chemical Society 124 (2002) 318-326. doi:10.1021/ja0118340.

[14] K. Gawrisch, N. V. Eldho, L. L. Holte, The structure of DHA in phospholipid membranes, Lipids 38 (2003) 445-452. doi:10.1007/s11745-003-1082-0.

[15] J. B. Klauda, V. Monje, T. Kim, W. Im, Improving the CHARMM Force Field for Polyunsaturated Fatty Acid Chains, The Journal of Physical Chemistry B 116 (2012) 9424-9431. doi:10.1021/jp304056p.

[16] N. Kučerka, M.-P. Nieh, J. Katsaras, Fluid phase lipid areas and bilayer thicknesses of commonly used phosphatidylcholines as a function of temperature, Biochimica et Biophysica Acta (BBA) - Biomembranes 1808 (2011) 2761-2771. doi:10.1016/j.bbamem.2011.07.022.

[17] T. A. Harroun, J. Katsaras, S. R. Wassall, Cholesterol Is Found To Reside in the Center of a Polyunsaturated Lipid Membrane, Biochemistry 47 (2008) 7090-7096. doi:10.1021/bi800123b.

[18] F. A. Heberle, R. S. Petruzielo, J. Pan, P. Drazba, N. Kučerka, R. F. Standaert, G. W. Feigenson, J. Katsaras, Bilayer Thickness Mismatch Controls Domain Size in Model Membranes, Journal of the American Chemical Society 135 (2013) 6853-6859. doi:10.1021/ja3113615.

[19] O. Kratky, H. Leopold, H. Stabinger, The determination of the partial specific volume of proteins by the mechanical oscillator technique., Methods in enzymology 27 (1973) 98-110. URL: http://www.ncbi.nlm.nih.gov/pubmed/4797943. doi:10.1016/s00766879(73)27007-6.

[20] A. Hodzic, M. Rappolt, H. Amenitsch, P. Laggner, G. Pabst, Differential Modulation of Membrane Structure and Fluctuations by Plant Sterols and Cholesterol, Biophysical Journal 94 (2008) 3935-3944. doi:10.1529/biophysj.107.123224. 
[21] J. Pan, F. A. Heberle, S. Tristram-Nagle, M. Szymanski, M. Koepfinger, J. Katsaras, N. Kučerka, Molecular structures of fluid phase phosphatidylglycerol bilayers as determined by small angle neutron and X-ray scattering, Biochimica et Biophysica Acta (BBA) - Biomembranes 1818 (2012) 2135-2148. doi:10.1016/j.bbamem.2012.05.007.

[22] O. Arnold, J. Bilheux, J. Borreguero, A. Buts, S. Campbell, L. Chapon, M. Doucet, N. Draper, R. Ferraz Leal, M. Gigg, V. Lynch, A. Markvardsen, D. Mikkelson, R. Mikkelson, R. Miller, K. Palmen, P. Parker, G. Passos, T. Perring, P. Peterson, S. Ren, M. Reuter, A. Savici, J. Taylor, R. Taylor, R. Tolchenov, W. Zhou, J. Zikovsky, Mantid-Data analysis and visualization package for neutron scattering and $\mu \mathrm{SR}$ experiments, Nuclear Instruments and Methods in Physics Research Section A: Accelerators, Spectrometers, Detectors and Associated Equipment 764 (2014) 156-166. doi:10.1016/j.nima.2014.07.029.

[23] S. Jo, J. B. Lim, J. B. Klauda, W. Im, CHARMM-GUI Membrane Builder for Mixed Bilayers and Its Application to Yeast Membranes, Biophysical Journal 97 (2009) 50-58. doi:10.1016/j.bpj.2009.04.013.

[24] J. C. Phillips, R. Braun, W. Wang, J. Gumbart, E. Tajkhorshid, E. Villa, C. Chipot, R. D. Skeel, L. Kalé, K. Schulten, Scalable molecular dynamics with NAMD, Journal of Computational Chemistry 26 (2005) 1781-1802. doi:10.1002/jcc.20289.

[25] J. B. Klauda, R. M. Venable, J. A. Freites, J. W. O'Connor, D. J. Tobias, C. MondragonRamirez, I. Vorobyov, A. D. MacKerell, R. W. Pastor, Update of the CHARMM AllAtom Additive Force Field for Lipids: Validation on Six Lipid Types, The Journal of Physical Chemistry B 114 (2010) 7830-7843. doi:10.1021/jp101759q.

[26] T. Darden, D. York, L. Pedersen, Particle mesh Ewald: An N $\log (\mathrm{N})$ method for Ewald sums in large systems, The Journal of Chemical Physics 98 (1993) 10089-10092. doi:10.1063/1.464397.

[27] M. Tuckerman, B. J. Berne, G. J. Martyna, Reversible multiple time scale molecular dynamics, The Journal of Chemical Physics 97 (1992) 1990-2001. doi:10.1063/1.463137. 
[28] J.-P. Ryckaert, G. Ciccotti, H. J. Berendsen, Numerical integration of the cartesian equations of motion of a system with constraints: molecular dynamics of n-alkanes, Journal of Computational Physics 23 (1977) 327-341. doi:10.1016/0021-9991(77)900985.

[29] G. J. Martyna, M. L. Klein, M. Tuckerman, Nosé-Hoover chains: The canonical ensemble via continuous dynamics, The Journal of Chemical Physics 97 (1992) 2635-2643. doi:10.1063/1.463940.

[30] S. E. Feller, Y. Zhang, R. W. Pastor, B. R. Brooks, Constant pressure molecular dynamics simulation: The Langevin piston method, The Journal of Chemical Physics 103 (1995) 4613-4621. doi:10.1063/1.470648.

[31] J. Sonne, F. Y. Hansen, G. H. Peters, Methodological problems in pressure profile calculations for lipid bilayers, The Journal of Chemical Physics 122 (2005) 124903. doi:10.1063/1.1862624.

[32] O. Berger, O. Edholm, F. Jähnig, Molecular dynamics simulations of a fluid bilayer of dipalmitoylphosphatidylcholine at full hydration, constant pressure, and constant temperature, Biophysical Journal 72 (1997) 2002-2013. doi:10.1016/S0006-3495(97)78845-3.

[33] A. R. Braun, J. N. Sachs, J. F. Nagle, Comparing Simulations of Lipid Bilayers to Scattering Data: The GROMOS 43A1-S3 Force Field, The Journal of Physical Chemistry В 117 (2013) 5065-5072. doi:10.1021/jp401718k.

[34] N. Kučerka, J. Katsaras, J. F. Nagle, Comparing membrane simulations to scattering experiments: introducing the SIMtoEXP software., The Journal of membrane biology 235 (2010) 43-50. doi:10.1007/s00232-010-9254-5.

[35] J. Pan, D. Marquardt, F. A. Heberle, N. Kučerka, J. Katsaras, Revisiting the bilayer structures of fluid phase phosphatidylglycerol lipids: Accounting for exchangeable hydrogens, Biochimica et Biophysica Acta (BBA) - Biomembranes 1838 (2014) 2966-2969. doi:10.1016/j.bbamem.2014.08.009. 
[36] J. Pan, X. Cheng, L. Monticelli, F. A. Heberle, N. Kučerka, D. P. Tieleman, J. Katsaras, The molecular structure of a phosphatidylserine bilayer determined by scattering and molecular dynamics simulations, Soft Matter 10 (2014) 3716. doi:10.1039/c4sm00066h.

[37] N. Kučerka, B. van Oosten, J. Pan, F. A. Heberle, T. A. Harroun, J. Katsaras, Molecular Structures of Fluid Phosphatidylethanolamine Bilayers Obtained from Simulation-toExperiment Comparisons and Experimental Scattering Density Profiles, The Journal of Physical Chemistry B 119 (2015) 1947-1956. doi:10.1021/jp511159q.

[38] J. F. Nagle, Introductory lecture: Basic quantities in model biomembranes, Faraday Discuss. 161 (2013) 11-29. doi:10.1039/C2FD20121F.

[39] Q. Waheed, O. Edholm, Undulation Contributions to the Area Compressibility in Lipid Bilayer Simulations, Biophysical Journal 97 (2009) 2754-2760. doi:10.1016/j.bpj.2009.08.048.

[40] W. Rawicz, K. Olbrich, T. McIntosh, D. Needham, E. Evans, Effect of Chain Length and Unsaturation on Elasticity of Lipid Bilayers, Biophysical Journal 79 (2000) 328-339. doi:10.1016/S0006-3495(00)76295-3.

[41] N. Kučerka, J. F. Nagle, J. N. Sachs, S. E. Feller, J. Pencer, A. Jackson, J. Katsaras, Lipid Bilayer Structure Determined by the Simultaneous Analysis of Neutron and X-Ray Scattering Data, Biophysical Journal 95 (2008) 2356-2367. doi:10.1529/biophysj.108.132662.

[42] N. Kučerka, J. Gallová, D. Uhríková, P. Balgavý, M. Bulacu, S.-J. Marrink, J. Katsaras, Areas of Monounsaturated Diacylphosphatidylcholines, Biophysical Journal 97 (2009) 1926-1932. doi:10.1016/j.bpj.2009.06.050.

[43] W.-J. Sun, R. M. Suter, M. A. Knewtson, C. R. Worthington, S. Tristram-Nagle, R. Zhang, J. F. Nagle, Order and disorder in fully hydrated unoriented bilayers of gel-phase dipalmitoylphosphatidylcholine, Physical Review E 49 (1994) 4665-4676. doi:10.1103/PhysRevE.49.4665. 
[44] J. F. Nagle, S. Tristram-Nagle, Structure of lipid bilayers, Biochimica et Biophysica Acta (BBA) - Reviews on Biomembranes 1469 (2000) 159-195. doi:10.1016/S03044157(00)00016-2.

[45] J. Huang, G. W. Feigenson, A Microscopic Interaction Model of Maximum Solubility of Cholesterol in Lipid Bilayers, Biophysical Journal 76 (1999) 2142-2157. doi:10.1016/S0006-3495(99)77369-8.

[46] J. F. Nagle, Experimentally determined tilt and bending moduli of singlecomponent lipid bilayers, Chemistry and Physics of Lipids 205 (2017) 18-24. doi:10.1016/j.chemphyslip.2017.04.006.

[47] H. I. Petrache, A. Salmon, M. F. Brown, Structural properties of docosahexaenoyl phospholipid bilayers investigated by solid-state $2 \mathrm{~h} \mathrm{nmr}$ spectroscopy, Journal of the American Chemical Society 123 (2001) 12611-12622. URL: https://doi.org/10.1021/ja011745n. doi:10.1021/ja011745n, pMID: 11741426.

[48] K. Rajamoorthi, H. I. Petrache, T. J. McIntosh, M. F. Brown, Packing and viscoelasticity of polyunsaturated -3 and -6 lipid bilayers as seen by $2 \mathrm{~h} \mathrm{nmr}$ and $\mathrm{x}$-ray diffraction, Journal of the American Chemical Society 127 (2005) 1576-1588. URL: https://doi .org/10.1021/ja046453b. doi:10.1021/ja046453b, pMID: 15686391.

[49] L. B. Li, I. Vorobyov, T. W. Allen, The role of membrane thickness in charged protein-lipid interactions, Biochimica et Biophysica Acta (BBA) - Biomembranes 1818 (2012) 135-145. doi:10.1016/j.bbamem.2011.10.026. 


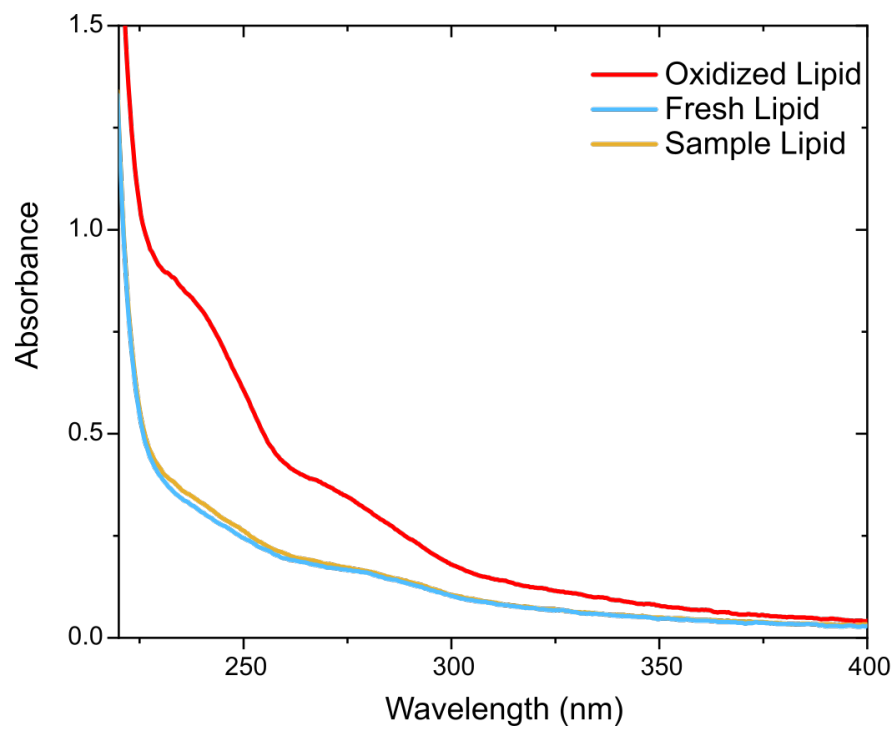

Figure 1: UV absorption spectra of SDPC bilayers composed of oxidized lipid (red line), freshly prepared lipid (blue), and a sealed sample studied by SANS approximately 12 hours after the measurement (orange line). The oxidized lipid was exposed to air for 12 hours. 
Table 1: SDP Structural Data of PDPC Bilayers

\begin{tabular}{|l|c|c|c|c|c|}
\hline & \multicolumn{4}{|c|}{ PDPC } & SDPC \\
\hline & SDP model & SDP model & SDP model & CHARMM & SDP model \\
\hline & $20^{\circ} \mathrm{C}$ & $30^{\circ} \mathrm{C}$ & $40^{\circ} \mathrm{C}$ & $30^{\circ} \mathrm{C}$ & $30^{\circ} \mathrm{C}$ \\
\hline$V_{L}\left(\AA^{3}\right)$ & $1296.6^{* *}$ & $1306.4^{* *}$ & $1316.1^{* *}$ & 1312.0 & $1366.4^{* *}$ \\
\hline$V_{H L}\left(\AA^{3}\right)$ & $320.0^{* *}$ & $320.0^{* *}$ & $320.0^{* *}$ & 320.0 & $320.0^{* *}$ \\
\hline$A\left(\AA^{2}\right)$ & 69.3 & 71.1 & 72.9 & 72.0 & 70.4 \\
\hline$D_{B}(\AA)$ & 37.4 & 36.8 & 36.1 & 36.4 & 38.8 \\
\hline$D_{H H}(\AA)$ & 33.2 & 33.0 & 32.2 & 36.8 & 35.2 \\
\hline $2 D_{C}(\AA)$ & 28.2 & 27.8 & 27.3 & 27.5 & 29.7 \\
\hline$D_{H 1}(\AA)$ & 2.51 & 2.63 & 2.43 & 4.63 & 2.73 \\
\hline$z_{G 1}(\AA)$ & 15.3 & 14.9 & 14.6 & 15.0 & 16.1 \\
\hline$\sigma_{G 1}(\AA)$ & 2.84 & 2.82 & 2.70 & 2.88 & 2.89 \\
\hline$z_{G 2}(\AA)$ & 16.7 & 17.0 & 16.6 & 19.0 & 17.7 \\
\hline$\sigma_{G 2}(\AA)$ & 3.23 & 3.30 & 3.37 & 2.95 & 3.14 \\
\hline$z_{G 3}(\AA)$ & $20.2^{*}$ & $20.0^{*}$ & $19.9^{*}$ & 20.5 & $20.9^{*}$ \\
\hline$\sigma \mathrm{G} 3(\AA)$ & $3.45^{*}$ & $3.44^{*}$ & $3.44^{*}$ & 3.46 & $3.46^{*}$ \\
\hline $\mathrm{z} \mathrm{CHb}(\AA)$ & 8.6 & 8.00 & 7.8 & 10.1 & 8.0 \\
\hline$\sigma \mathrm{CHb}(\AA)$ & $3.52^{*}$ & $3.52^{*}$ & $3.52^{*}$ & 3.50 & $3.51^{*}$ \\
\hline$z \mathrm{CHa}(\AA)$ & 2.8 & 2.2 & 2.0 & 4.3 & 2.3 \\
\hline$\sigma \mathrm{CHa}(\AA)$ & $4.08^{*}$ & $4.07^{*}$ & $4.07^{*}$ & 4.1 & 4.08 \\
\hline$z_{C H b}-z_{C H a}(\AA)$ & $5.8^{*}$ & $5.8^{*}$ & $5.8^{*}$ & 5.8 & $5.7^{*}$ \\
\hline$\sigma_{H C}(\AA)$ & $2.84^{*}$ & $2.84^{*}$ & $2.83^{*}$ & 2.83 & $2.82^{*}$ \\
\hline$\sigma_{C H 3}(\AA)$ & $3.70^{*}$ & $3.70^{*}$ & $3.70^{*}$ & 4.20 & $3.88^{*}$ \\
\hline$r_{G 1}$ & $0.45^{*}$ & $0.44^{*}$ & $0.44^{*}$ & 0.45 & $0.45^{*}$ \\
\hline$r_{G 2}$ & $0.36^{*}$ & $0.36^{*}$ & $0.36^{*}$ & 0.36 & $0.36^{*}$ \\
\hline$r_{C H 3}$ & $2.02^{*}$ & $2.02^{*}$ & $2.02^{*}$ & 1.97 & $2.01^{*}$ \\
\hline$r_{C H}$ & $0.92^{*}$ & $0.92^{*}$ & $0.92^{*}$ & 0.92 & $0.92^{*}$ \\
\hline $\mathrm{P}^{*}$ & +1 & & & & \\
\hline
\end{tabular}

Parameters are shown with their appropriate units for length $(\AA)$, area $\left(\AA^{2}\right)$, and volume $\left(\AA^{3}\right)$. Estimated uncertainties are $\pm 2 \%$. The double asterisk $(* *)$ denotes fixed parameters, while a single asterisk $\left(^{*}\right)$ denotes "soft" constrained parameters, allowed to vary within limits. 


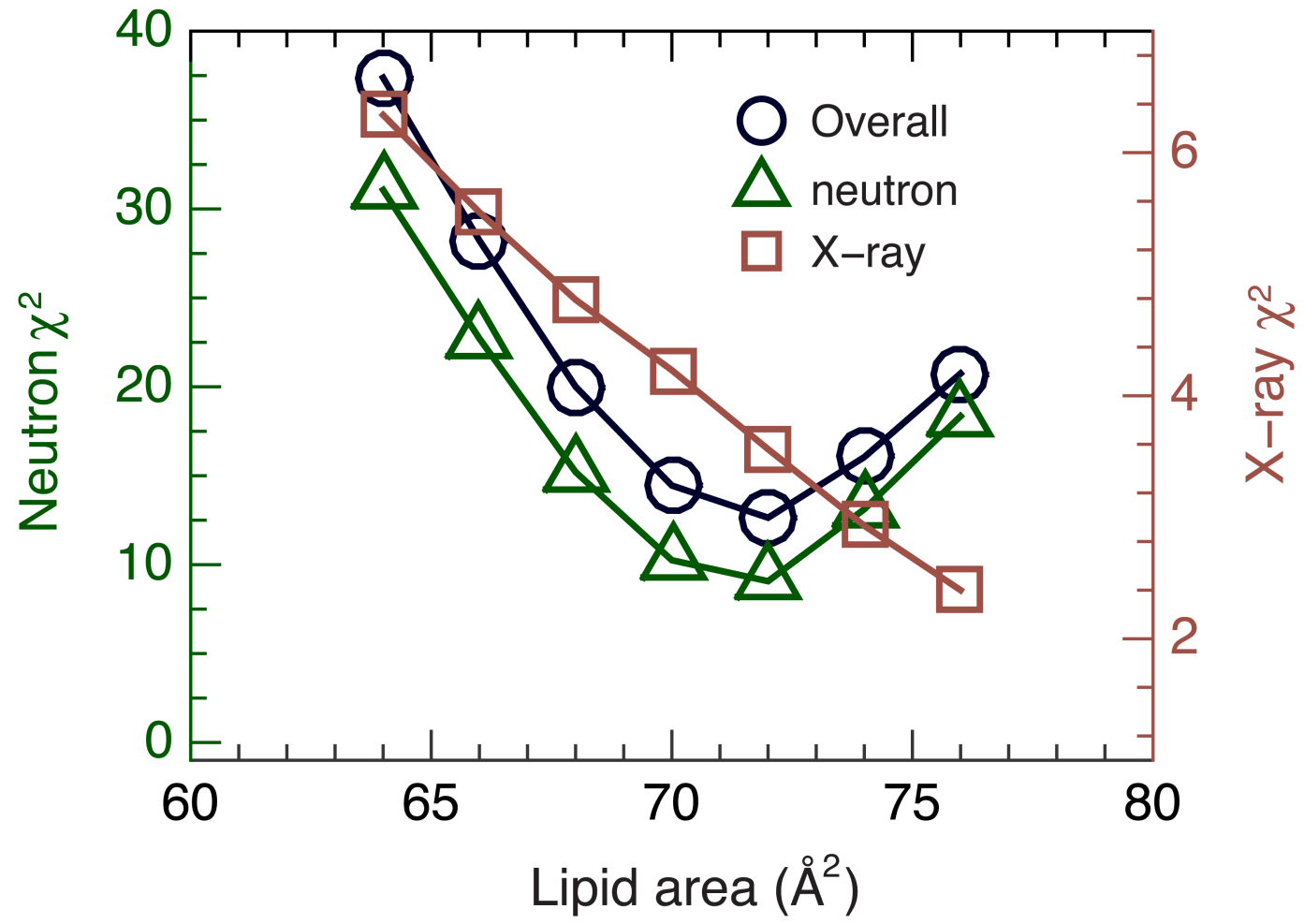

Figure 2: Model-free comparisons between experimental and simulated form factors for predetermined PDPC lipid areas. The neutron $\chi^{2}$ (open triangle, left axis) was obtained by summing the squares of the neutron form factor differences at $3 \mathrm{D}_{2} \mathrm{O}$ contrasts weighted by their experimental uncertainties and number of data points. The X-ray $\chi^{2}$ (open square, right axis) was obtained in a similar manner except that there is only one set of X-ray data. The overall $\chi^{2}$ (open circle, left axis) is a combination of neutron and X-ray $\chi^{2}$ 

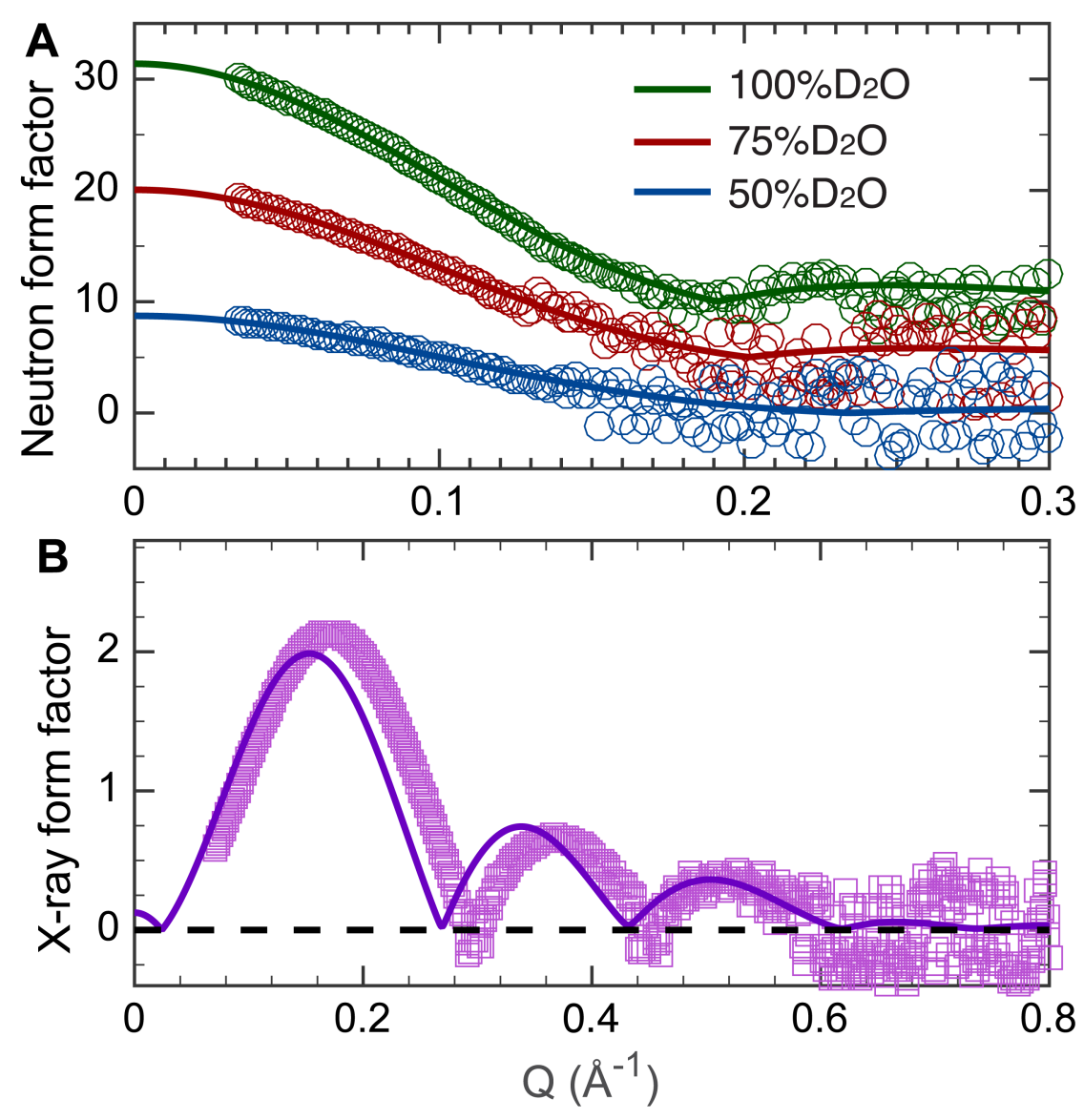

Figure 3: Model-free comparison between experimental scattering data and a simulated PDPC bilayer with an area per lipid constrained to $72.0 \AA^{2}$. The different contrast experimental (A) neutron and (B) X-ray form factors (symbols) are the same as those used in the SDP model analysis. The corresponding simulation form factors (solid lines) were calculated from atom number density distributions obtained from $N A P n T$ simulations. Each experimental form factor was scaled by a coefficient to minimize the reduced $\chi^{2}$, after taking experimental uncertainties into account. 


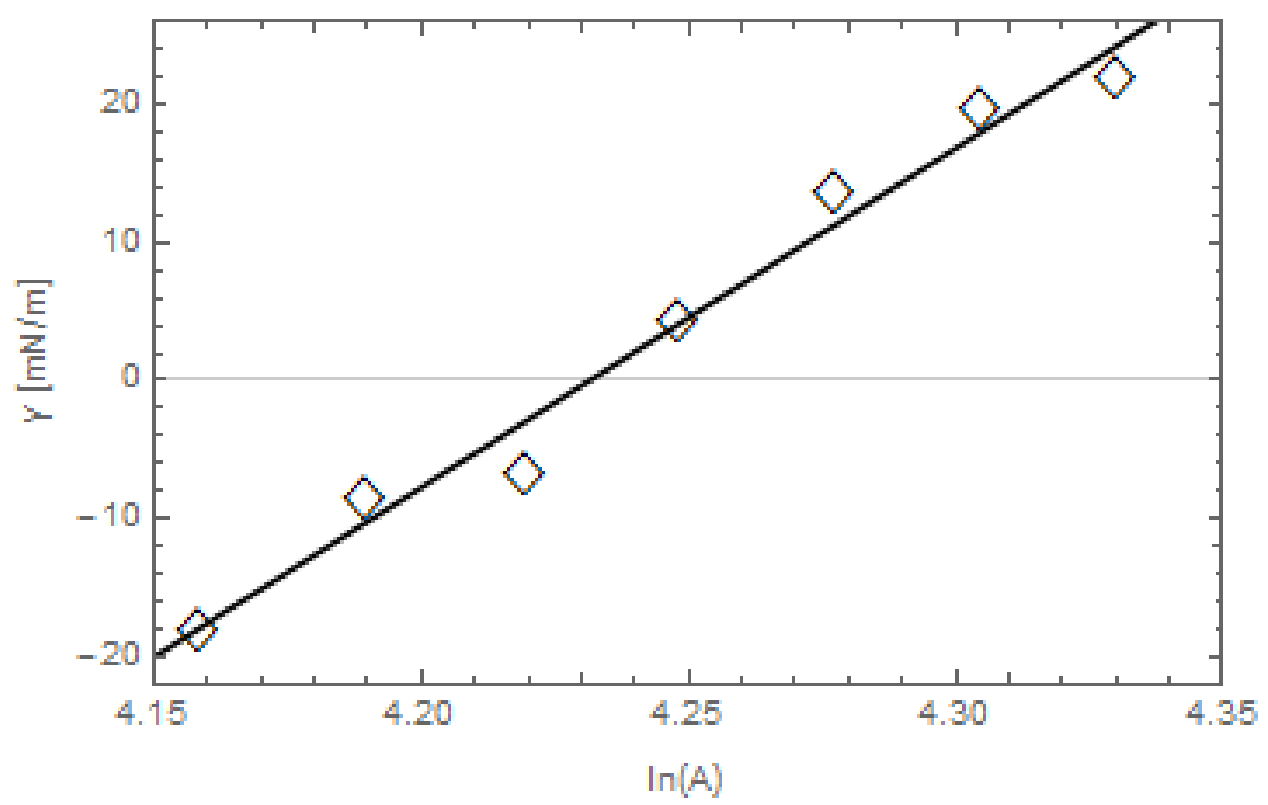

Figure 4: Lateral surface tension applied to a PDPC bilayer as a function of lipid area (A). The linear fit to the data (solid line) resulted in a calculated area compressibility modulus of $K_{A}=246.4 \mathrm{mN} / \mathrm{m}$. 

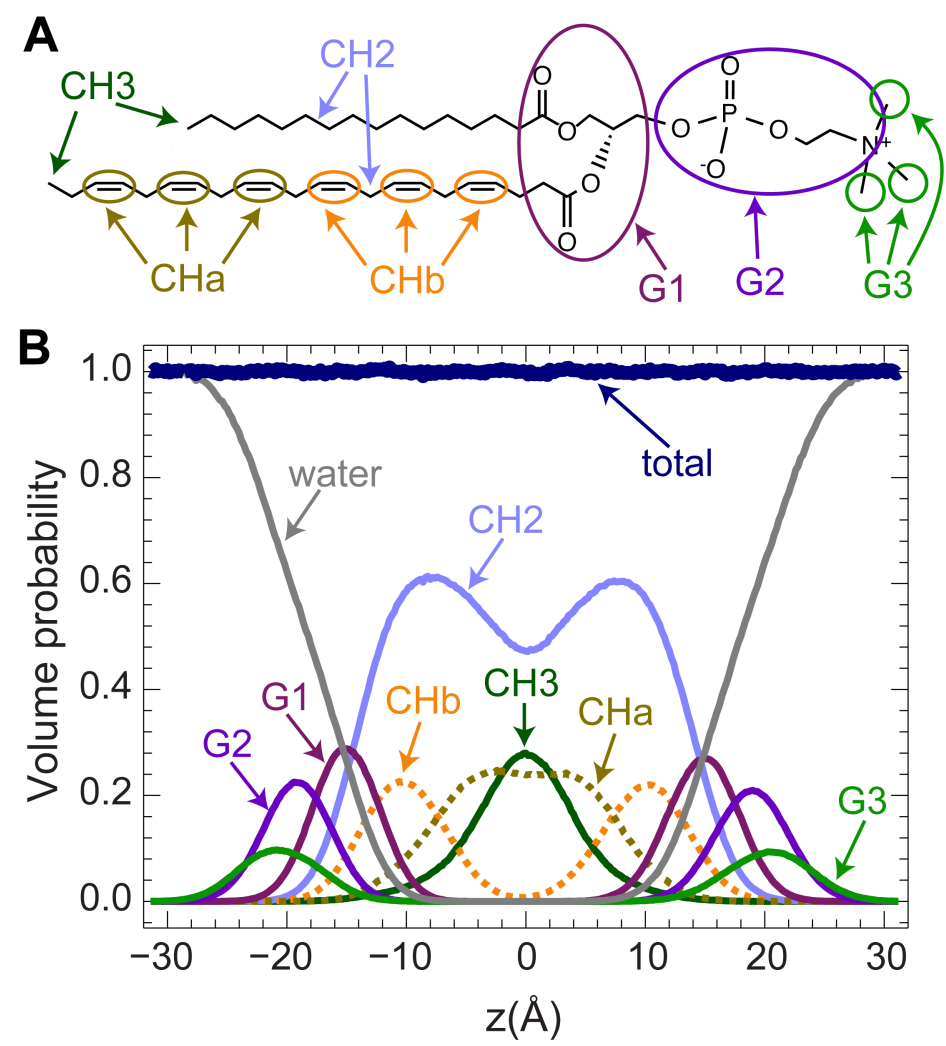

Figure 5: Parsing scheme for a PDPC bilayer based on MD simulations. A) The chemical structure of PDPC with different moieties is highlighted. B) The volume probabilities of each component based on the parsing scheme. Fatty acid chains were divided into terminal methyl $\left(\mathrm{CH}_{3}\right)$, methylene $\left(\mathrm{CH}_{2}\right)$ and methine $(\mathrm{CH})$, with double bonds grouped together as $\mathrm{CHa}$ and $\mathrm{CHb}$. The $\mathrm{PC}$ headgroup was parsed into the carbonyl-glycerol (G1), phosphate+CH2CH2N (G2) and the choline methyls (G3). 

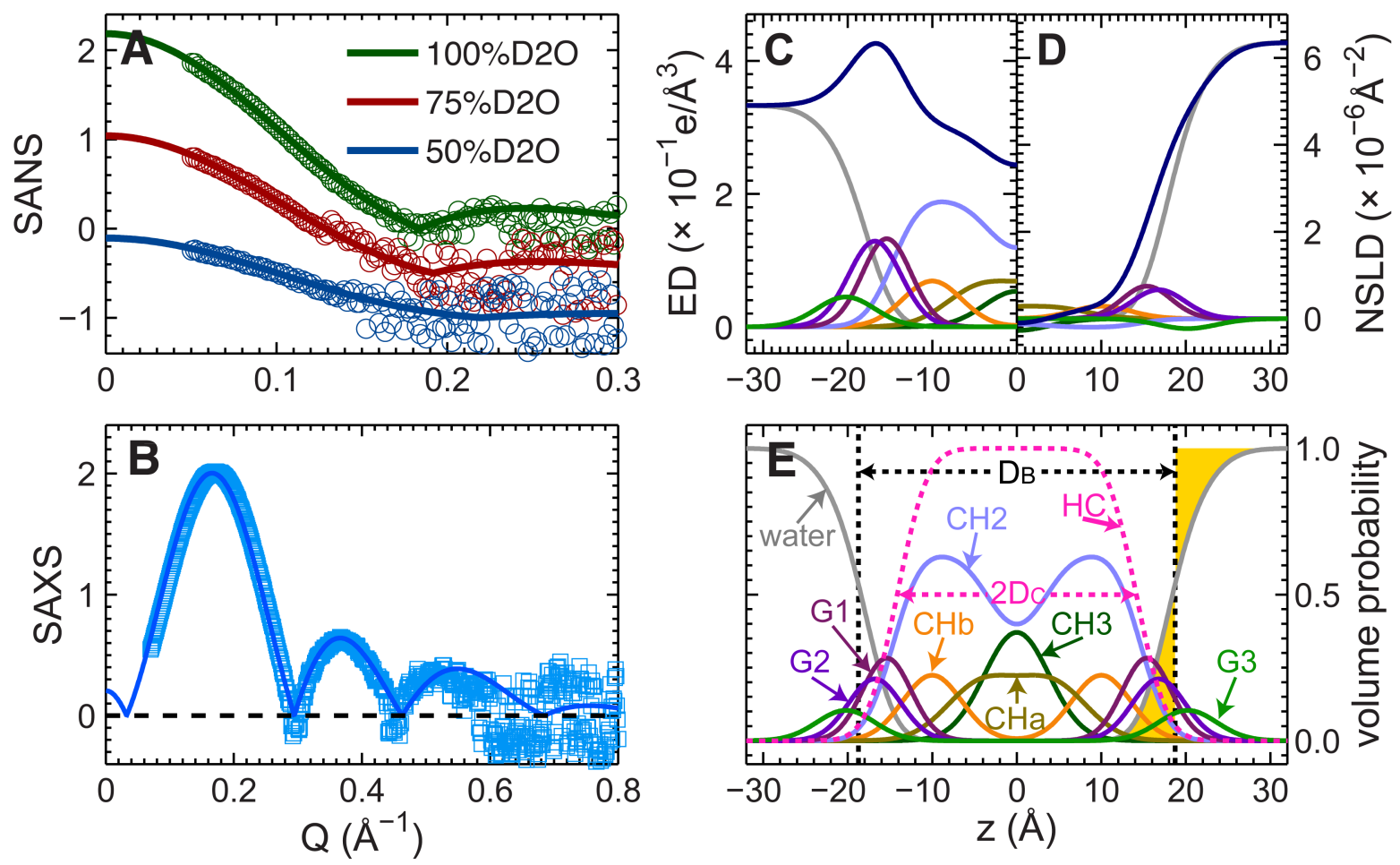

Figure 6: Fits (solid lines) to the experimental SANS (A) and SAXS (B) form factors (points) for PDPC at $30{ }^{\circ} \mathrm{C}$ using SDP. Panels on the right show the SDP model of the PDPC bilayer in real space, where the top panels show electron densities (C) and neutron scattering length densities (D) of the components making up the bilayer, including the total scattering densities (thick lines). (E)Bottom panel illustrates the volume probability distributions, where the total probability is equal to 1 at each point along the bilayer normal. 